\title{
Predictive Model for Leachate Contamination of Groundwater around a municipal Waste Dump: Case Study of Obosi, Southeastern Nigeria
}

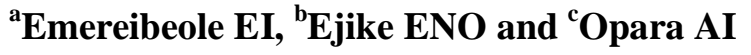 \\ ${ }^{a}$ Department of Environmental Management, Federal University of Technology, Owerri, Nigeria. \\ ${ }^{\mathrm{b}}$ Department of Chemistry, Federal University of Technology, Owerri, Nigeria. \\ ${ }^{\mathrm{c}}$ Department of Geology, Federal University of Technology, Owerri, Nigeria.
}

Correspondence Author: Emereibeole EI, Department of Environmental Management, Federal University of Technology, Owerri, Nigeria.

E-mail:- emereibeolee@yahoo.com

Received date: 18May 2019, Accepted date: 26 June 2019, Online date: 20 June 2019

Copyright: () 2019Emereibeole EI et al, This is an open-access article distributed under the terms of the Creative Commons Attribution License, which permits unrestricted use, distribution, and reproduction in any medium, provided the original author and source are credited.

\begin{abstract}
Landfill wastes undergo several decomposition processes giving rise to various organic and inorganic contaminants that have high toxic implications. These contaminants later dissolve in rain water to form leachate. The leachate in such waste streams could migrate through the porous soil media of the vadose region by several physical processes to contaminate the groundwater of the area. Leachate migration from waste sites to the groundwater is subject to various transport attributes such as hydraulic head, distance of borehole from the waste dump, borehole depth, net recharge of groundwater and leachate concentration. Space and time dependent pollution of groundwaters was predicted by developing a numerical model of polynomial expression which incorporates these transport attributes. The groundwater pollution was conceived to be a function of distance from dumpsite, $x_{1}$, porosity and conductivity of vadose region, $x_{2}$, depth of borehole, $x_{3}$, hydraulic head, $x_{4}$ and net recharge of groundwater, $x_{5}$. This concept was captured in the expression $y=k+k_{1} x_{1}+k_{2} x_{2}+k_{3} x_{3}+k_{4} x_{4}+k_{5} x_{5}$, where $y$ is the composite pollution index. The polynomial was solved by application of matrices algebra embedded in Matlab 7.9. The resulting prediction equation was tested for accuracy by comparison with cation analysis of four boreholes chosen at random within the study area. The one-way ANOVA test revealed that the mean concentrations of all the cations measured differed significantly across the boreholes studied ( $\mathrm{Sig}$ F values $=\mathrm{As} 0.005, \mathrm{~Pb} 0.000, \mathrm{Cu}$ 0.000, Cr 0.000, Co 0.000, Ni 0.000, Mn 0.037, Fe 0.000, Cd 0.000 and $\mathrm{Hg} \mathrm{0.000)}$ at $\mathrm{p}<0.05$ whereas the pair-wise student's t-test show that except for $\mathrm{Hg}$, the simulated and measured values of the other cations studied were not significantly different at the $95 \%$ confidence level (sig. $t=0.026$ ). This result indicates that at $95 \%$ confidence level, the simulation becomes invalid.
\end{abstract}

Keywords: prediction, waste dump, Southeastern Nigeria, trace metals, leachate

\section{INTRODUCTION}

Open wastes dumping and use of unprotected landfills for solid wastes disposal have been identified as the oldest ways of solid wastes disposal (Pariatamby and Tanaka, 2013). The use of unprotected landfills represents the most viable and commonly used method for solid waste disposal all over the world. This is because it may achieve the reclamation of derelict land (Erses et al., 2005). However, this practice is considered one of the major threats to groundwater (Fatta et al., 1999) and the scale of this threat depends on the concentration and toxicity of contaminants in the waste's leachate, type and permeability of geological strata, depth of water table and the direction of groundwater flow (Al-Khaldi, 2006). Point sources such as open waste dumps can release high concentrations of contaminants into the groundwater. The leachate generated primarily as a result of precipitation and biodegradation activities on the waste components have the potential to pollute the underlying groundwater aquifers (Jhnamnani and Singh, 2009), and impair its uses (Renou et al., 2008). There is a greater possibility of groundwater contamination in areas near open waste dumps and unprotected landfills due to the potential of such facilities to leak leachate originating from them. Such contamination of groundwater resource poses a substantial risk to public health and to the natural environment. Leachate migration from wastes sites or landfills to groundwater is subject to various processes such as weathering, solubility of the waste components, ion exchange and vadose region characteristics. Such characteristics are expressed by porosity and conductivity of 
the vadose region, rock formation and disposition, ion concentration and migration impedance. It equally depend on waste generation rate, wastes type and disposal methods. Many approaches have been adopted for the assessment of the impact of leachate seepage on groundwater. This can be achieved either by the experimental determination of the impurities or through mathematical modeling (Moo-Young et al., 2004, Hudak, 1998 and Stoline et al., 1993). A predictive model for leachate contamination of groundwater aims at predicting the tendency or likelihood for contaminants to reach a specified level or position in the groundwater system after introduction at some location above the uppermost aquifer (Thirumalaivasan, 2001). Obosi in Anambra State has experienced high rates of urbanization and industrialization. This has resulted to environmental challenges consequent on urbanization and industrialization involving population migration. The increase in population is associated with increased volume of generated wastes. The Obosi waste dumpsite is unprotected and has received wastes streams for over 50 years. The leachate in such waste streams could migrate through the porous soil media of the vadose region. Several physical processes leading to contamination of the shallow aquifers of the area occur. In recent times, the impact of leachate on groundwater sources has been extensively studied (Saarela, 2001; Abu and Kofahi, 2001; Christensen et al., 2001; De Rosa et al., 1996 and Flyhammer, 1995). Many researchers have also carried out investigations on the state of groundwater within Anambra state. Most of these studies are limited to spot measurements used for baseline studies (Egboka et al. 1983; Ezeabasili et al. 2015; Ezeabasili et al. 2014; Ogbukagu, 1986; etc). However, none of these studies proposed or used any simulation model capable of modeling leachate contamination pathways and dynamics. They also failed to link groundwater contamination in the area to environmental and hydro-geological attributes such as distance of borehole from the waste dump, porosity and hydraulic conductivity of the vadose region, borehole depth, net recharge of the aquifer and hydraulic head. If leachate contamination of groundwater around waste dumps can be accurately predicted, control and remediation measures can be put in place for adequate waste management. This study therefore presents predictive mathematical model of groundwater pollutant concentrations as a function of contaminant transport attributes. This will serve as a tool to guarantees clean water delivery (Salami et al., 2013).

\section{MATERIALS AND METHODS}

Field sampling was carried out using standard methods as provided by APHA/AWWA/WEF (1999). All physico-chemical parameters were determined based on National Environmental Standards and Regulations 2009 while heavy metals were determined using FS 240 Varian AAS. The analog map of the study area was digitized and delineated into 8 map groups with each group consisting of a series of concentric cells separated $500 \mathrm{~m}$ apart with borehole positions geo-referenced therein. Twenty four (24) boreholes located within $4 \mathrm{~km}$ radius around the Obosi dumpsite constituted the composite sampling locations and the waste dump was the centre of the study cells. Nine (9) trace metals were analysed for in each borehole for two different seasons. Results of analysis from the sampled boreholes were inserted into polynomial equations which were solved simultaneously using Matrix algebra, executed in Matlab 7.2 to arrive at predictive models.

Statistical Analysis: SPSS 17.0 statistical software was used for statistical analysis of data. ANOVA was used to establish the spatial variation in pollutant concentrations between the studied boreholes whereas the student's t-tests analysis was used to conduct a pair wise comparison between the simulated and field measurements.

\section{HYPOTHESIS OF THE STUDY}

The theory underlying this study consists in the assumption that pollutant concentration in borehole waters which are contiguous depend on such attributes as distance of borehole from the waste dump, hydraulic conductivity of the porous medium, borehole depth, net recharge rate and hydraulic head. This can be represented as

$\mathrm{y}=\mathrm{k}+\mathrm{k}_{1} \mathrm{x}_{1}+\mathrm{k}_{2} \mathrm{x}_{2}+\mathrm{k}_{3} \mathrm{x}_{3}+\mathrm{k}_{4} \mathrm{x}_{4}+\mathrm{k}_{5} \mathrm{x}_{5}$

where $\mathrm{y}$ is the extent of pollution in a cell contributed by attributes $\mathrm{x}_{1}$ to $\mathrm{x}_{5}$ and $\mathrm{k}$ 's are predictive constants.

If therefore, a concentric circle of eight cells centered at the dump site defined by geographical/spatial coordinates is taken in a group and the cells, defined as $1,2,3 \ldots 8$, can be identified uniquely, then for a group of bore holes in cells $1,2,3 \ldots 8$, we may write the polynomial equations for contaminant concentrations $\mathrm{y}_{1}, \mathrm{y}_{2}, \mathrm{y}_{3}, \ldots, \mathrm{y}_{8}$ as follows:

$\mathrm{y}_{1}=\mathrm{k}_{1}+\mathrm{k}_{11} \mathrm{x}_{11}+\mathrm{k}_{12} \mathrm{x}_{12}+\mathrm{k}_{13} \mathrm{x}_{13}+\mathrm{k}_{14} \mathrm{x}_{14}+\mathrm{k}_{15} \mathrm{x}_{15}$

$\mathrm{y}_{2}=\mathrm{k}_{1}+\mathrm{k}_{11} \mathrm{x}_{21}+\mathrm{k}_{12} \mathrm{x}_{22}+\mathrm{k}_{13} \mathrm{x}_{23}+\mathrm{k}_{14} \mathrm{x}_{24}+\mathrm{k}_{15} \mathrm{x}_{25}$

$\mathrm{y}_{3}=\mathrm{k}_{1}+\mathrm{k}_{11} \mathrm{x}_{31}+\mathrm{k}_{12} \mathrm{x}_{32}+\mathrm{k}_{13} \mathrm{x}_{33}+\mathrm{k}_{14} \mathrm{x}_{34}+\mathrm{k}_{15} \mathrm{x}_{35}$

$\mathrm{y}_{4}=\mathrm{k}_{1}+\mathrm{k}_{11} \mathrm{x}_{41}+\mathrm{k}_{12} \mathrm{x}_{42}+\mathrm{k}_{13} \mathrm{x}_{43}+\mathrm{k}_{14} \mathrm{x}_{44}+\mathrm{k}_{15} \mathrm{x}_{45}$

$\mathrm{y}_{5}=\mathrm{k}_{1}+\mathrm{k}_{11} \mathrm{x}_{51}+\mathrm{k}_{12} \mathrm{x}_{52}+\mathrm{k}_{13} \mathrm{x}_{53}+\mathrm{k}_{14} \mathrm{x}_{54}+\mathrm{k}_{15} \mathrm{x}_{55}$

$\mathrm{y}_{6}=\mathrm{k}_{1}+\mathrm{k}_{11} \mathrm{x}_{61}+\mathrm{k}_{12} \mathrm{x}_{62}+\mathrm{k}_{13} \mathrm{x}_{63}+\mathrm{k}_{14} \mathrm{x}_{64}+\mathrm{k}_{15} \mathrm{x}_{65}$

$\mathrm{y}_{7}=\mathrm{k}_{1}+\mathrm{k}_{11} \mathrm{x}_{71}+\mathrm{k}_{12} \mathrm{x}_{72}+\mathrm{k}_{13} \mathrm{x}_{73}+\mathrm{k}_{14} \mathrm{x}_{74}+\mathrm{k}_{15} \mathrm{x}_{75}$

$\mathrm{y}_{8}=\mathrm{k}_{1}+\mathrm{k}_{11} \mathrm{x}_{81}+\mathrm{k}_{12} \mathrm{x}_{82}+\mathrm{k}_{13} \mathrm{x}_{83}+\mathrm{k}_{14} \mathrm{x}_{84}+\mathrm{k}_{15} \mathrm{x}_{85}$

The equations can be solved by applying matrix algebra as follows: 
Citation: Emereibeole EI, et al., Predictive Model for Leachate Contamination of Groundwater around a municipal Waste Dump: Case Study of Obosi, Southeastern Nigeria. Australian Journal of Basic and Applied Sciences, 13(6): 115-121.DOI: 10.22587/ajbas.2019.13.6.13

$\left(\begin{array}{l}\mathrm{y}_{1} \\ \mathrm{y}_{2} \\ \mathrm{y}_{3} \\ \mathrm{y}_{4} \\ \mathrm{y}_{5} \\ \mathrm{y}_{6} \\ \mathrm{y}_{7} \\ \mathrm{y}_{8}\end{array}\right)=\left(\begin{array}{cccccc}1 & \mathrm{x}_{11} & \mathrm{x}_{12} & \mathrm{x}_{13} & \mathrm{x}_{14} & \mathrm{x}_{15} \\ 1 & \mathrm{x}_{21} & \mathrm{x}_{22} & \mathrm{x}_{23} & \mathrm{x}_{24} & \mathrm{x}_{25} \\ 1 & \mathrm{x}_{31} & \mathrm{x}_{32} & \mathrm{x}_{33} & \mathrm{x}_{34} & \mathrm{x}_{35} \\ 1 & \mathrm{x}_{41} & \mathrm{x}_{42} & \mathrm{x}_{43} & \mathrm{x}_{44} & \mathrm{x}_{45} \\ 1 & \mathrm{x}_{51} & \mathrm{x}_{52} & \mathrm{x}_{53} & \mathrm{x}_{54} & \mathrm{x}_{55} \\ 1 & \mathrm{x}_{61} & \mathrm{x}_{62} & \mathrm{x}_{63} & \mathrm{x}_{64} & \mathrm{x}_{65} \\ 1 & \mathrm{x}_{71} & \mathrm{x}_{72} & \mathrm{x}_{73} & \mathrm{x}_{74} & \mathrm{x}_{75} \\ 1 & \mathrm{x}_{81} & \mathrm{x}_{82} & \mathrm{x}_{83} & \mathrm{x}_{84} & \mathrm{x}_{85}\end{array}\right)\left(\begin{array}{l}\mathrm{k}_{1} \\ \mathrm{k}_{11} \\ \mathrm{k}_{12} \\ \mathrm{k}_{13} \\ \mathrm{k}_{14} \\ \mathrm{k}_{15} \\ \mathrm{k}_{16} \\ \mathrm{k}_{17}\end{array}\right)$

The solution to this matrix using MATLAB 7.2 gives the k's which are expected to be uniquely constant in a given cell and for a given pollutant parameter for any bore hole in the cell. therein.

Figure 1 represents digitized map of the study area delineated into eight concentric study cells and the boreholes contained

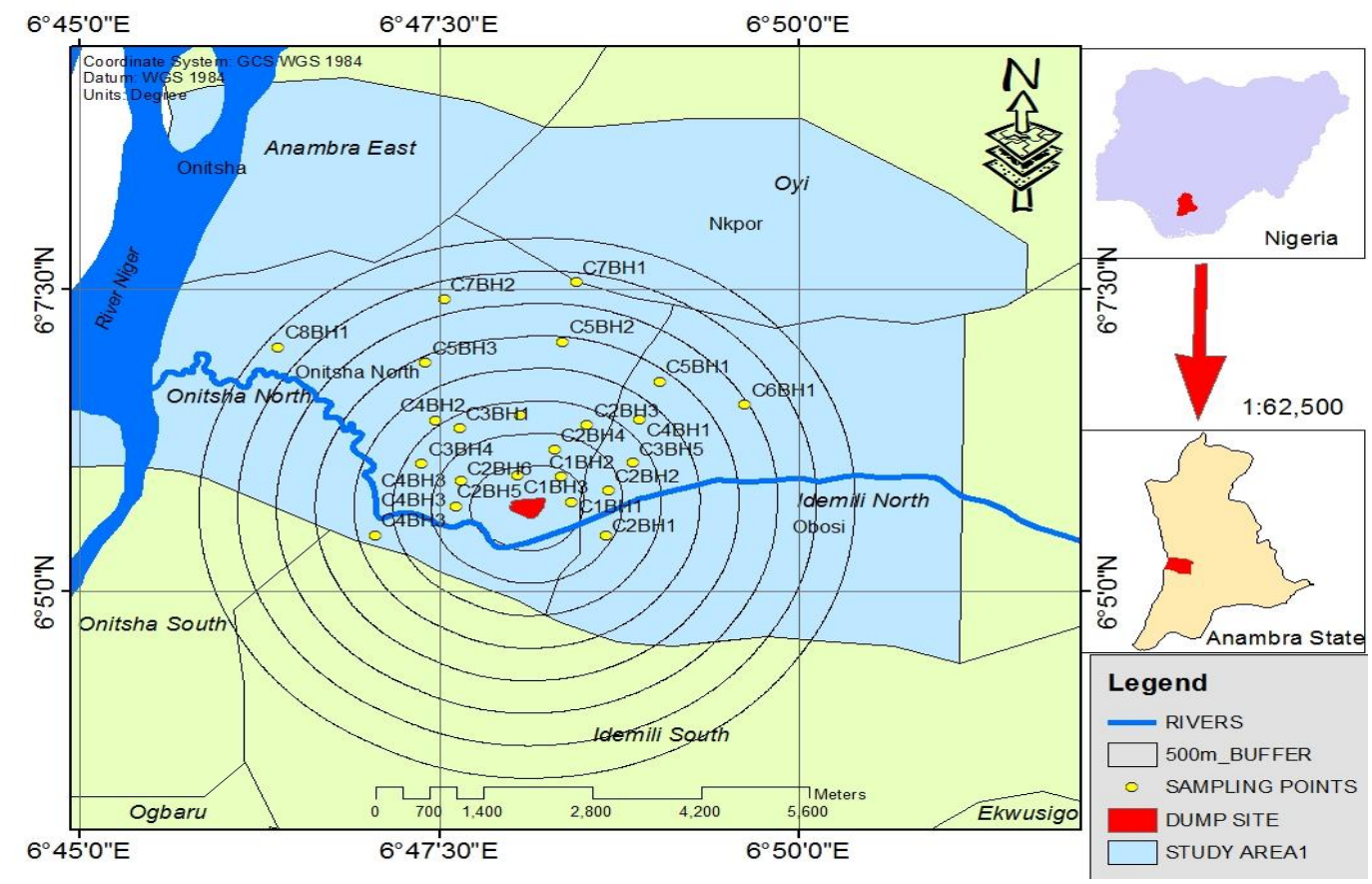

Figure 1: Map of study area showing the study cells and sampling points

\section{RESULTS}

Tables 1 Show the trace metal levels obtained from the laboratory analysis of water samples in wet and dry seasons.

Table 1: Levels of trace metals in groundwater around the Obosi dumpsite (wet and dry seasons).

\begin{tabular}{|c|c|c|c|c|c|c|c|c|c|c|c|c|c|c|c|c|c|c|c|c|}
\hline $\begin{array}{l}\text { SAM } \\
\text { PLE }\end{array}$ & $\begin{array}{c}\text { LO } \\
\text { NG. } \\
\text { ( E) }\end{array}$ & $\begin{array}{c}\text { LA } \\
\mathbf{T} \\
(\mathbf{N})\end{array}$ & $\begin{array}{c}\mathrm{Pb} \\
\mathrm{mg} \\
/ \mathrm{L}\end{array}$ & $\begin{array}{c}\mathrm{Cu} \\
\mathrm{mg} \\
/ \mathrm{L}\end{array}$ & $\begin{array}{c}\mathrm{Cr} \\
\mathrm{mg} \\
/ \mathrm{L}\end{array}$ & $\begin{array}{c}\text { Co } \\
\text { mg } \\
/ \mathrm{L}\end{array}$ & $\begin{array}{c}\text { Ni } \\
\mathbf{m g} \\
/ \mathrm{L}\end{array}$ & $\begin{array}{c}\text { M } \\
\mathbf{n} \\
\mathbf{m g} \\
/ \mathrm{L}\end{array}$ & $\begin{array}{l}\mathrm{Fe} \\
\mathrm{mg} \\
/ \mathrm{L}\end{array}$ & $\begin{array}{c}\text { Cd } \\
\text { mg } \\
/ \mathrm{L}\end{array}$ & $\begin{array}{c}\mathrm{Hg} \\
\mathrm{mg} \\
/ \mathrm{L}\end{array}$ & $\begin{array}{c}\mathrm{Pb} \\
\mathrm{mg} \\
/ \mathrm{L}\end{array}$ & $\begin{array}{c}\mathrm{Cu} \\
\mathbf{m g} \\
/ \mathrm{L}\end{array}$ & $\begin{array}{c}\mathrm{Cr} \\
\mathrm{mg} \\
/ \mathrm{L}\end{array}$ & $\begin{array}{c}\text { Co } \\
\text { mg } \\
/ \mathrm{L}\end{array}$ & $\begin{array}{c}\mathrm{Ni} \\
\mathbf{m g} \\
/ \mathrm{L}\end{array}$ & $\begin{array}{c}\text { M } \\
\mathbf{n} \\
\mathbf{m g} \\
/ \mathrm{L}\end{array}$ & $\begin{array}{c}\mathrm{Fe} \\
\mathrm{mg} \\
/ \mathrm{L}\end{array}$ & $\begin{array}{c}\text { Cd } \\
\text { mg } \\
/ \mathrm{L}\end{array}$ & $\begin{array}{c}\mathrm{Hg} \\
\mathrm{mg} \\
/ \mathrm{L}\end{array}$ \\
\hline & & & \multicolumn{9}{|c|}{ WET SEASON } & \multicolumn{9}{|c|}{ DRY SEASON } \\
\hline $\mathrm{C}_{1} \mathrm{BH}$ & 6.81 & 6.0 & 0.0 & 0.0 & 0.0 & 0.0 & 0.0 & 0.0 & 0.0 & 0.0 & 2.2 & 0.4 & 0.0 & 1.0 & 0.3 & 0.0 & 0.2 & 0.0 & 0.0 & 1.1 \\
\hline 1 & 10 & 970 & 7 & 29 & 00 & 00 & 00 & 00 & 00 & 00 & 00 & 09 & 15 & 42 & 11 & 00 & 94 & 00 & 55 & 06 \\
\hline
\end{tabular}


Citation: Emereibeole EI, et al., Predictive Model for Leachate Contamination of Groundwater around a municipal Waste Dump: Case Study of Obosi, Southeastern Nigeria. Australian Journal of Basic and Applied Sciences, 13(6): 115-121.DOI: 10.22587/ajbas.2019.13.6.13

\begin{tabular}{|c|c|c|c|c|c|c|c|c|c|c|c|c|c|c|c|c|c|c|c|c|}
\hline BH & $\begin{array}{l}5.81 \\
40\end{array}$ & $\begin{array}{c}6.1 \\
010\end{array}$ & $\begin{array}{l}2^{1} \\
\end{array}$ & $\begin{array}{c}0.0 \\
07\end{array}$ & $\begin{array}{c}0.0 \\
00\end{array}$ & $\begin{array}{c}0.0 \\
00\end{array}$ & $\begin{array}{c}0.0 \\
00\end{array}$ & $\begin{array}{c}0.0 \\
00\end{array}$ & $\begin{array}{l}0.2 \\
24\end{array}$ & $\begin{array}{c}0.2 \\
04\end{array}$ & 0 & $\begin{array}{c}0.1 \\
20\end{array}$ & $\begin{array}{c}0.0 \\
07\end{array}$ & $\begin{array}{l}0.0 \\
00\end{array}$ & $\begin{array}{l}0 \\
0\end{array}$ & $\begin{array}{c}0.0 \\
00\end{array}$ & $\begin{array}{c}0.2 \\
00\end{array}$ & $\begin{array}{l}0.2 \\
24\end{array}$ & $\begin{array}{l}0.2 \\
04\end{array}$ & $\begin{array}{l}0.2 \\
36 \\
\end{array}$ \\
\hline $\mathrm{C}_{1} \mathrm{BH}$ & 6.80 & & & & $\begin{array}{c}0.0 \\
00\end{array}$ & $\begin{array}{c}0.0 \\
00\end{array}$ & 0.0 & 0 & 0.3 & & & 0.4 & 0.0 & .1 & 4 & $\begin{array}{l}0.1 \\
14\end{array}$ & .6 & & 0.0 & .2 \\
\hline $\mathrm{C}_{2} \mathrm{BH}$ & & & & & 2.0 & 2.9 & 0.1 & & 2.0 & 0.0 & & & & & & 0.0 & 9 & & & 2 \\
\hline $\mathrm{C}_{2} \mathrm{BH}$ & & & & & 0 &.( & & .8 & 0 & 0 & & & 0. & & .1 & .0 & & & & .2 \\
\hline $\mathrm{C}_{2} \mathrm{BH}$ & & & & & & & & & & & & & & & & & & & & .1 \\
\hline $\mathrm{C}_{2} \mathrm{BH}$ & & & & & & $\begin{array}{l}0.1 \\
15\end{array}$ & 0.0 & 54 & 75 & & & & & & 0 & 67 & & & & .1 \\
\hline $\mathrm{C}_{2} \mathrm{BH}$ & & & & .0 & $\begin{array}{c}0.0 \\
00\end{array}$ & & $\begin{array}{c}0.0 \\
00\end{array}$ & $\begin{array}{l}0.0 \\
00\end{array}$ & & & & & & & .1 & 0.0 & & & & \\
\hline $\mathrm{C}_{2} \mathrm{BH}$ & & & & 00 & & & & 00 & & & & & & & & & & & & .1 \\
\hline $\mathrm{C}_{3} \mathrm{BH}$ & & & & & & & & 1.7 & & & & & $\begin{array}{l}0 . \\
00\end{array}$ & & .0 & 0.0 & 2 & & & 0.0 \\
\hline & & & & 07 & & & & 74 & & & 00 & & & & & & & & & .1 \\
\hline $\mathrm{C}_{3} \mathrm{BH}$ & & & & & & $\begin{array}{c}0.0 \\
09\end{array}$ & & 0.0 & & & & & & & & 0.0 & & & & 0.1 \\
\hline & & & & & & & & & & & & & & & & & & & & .0 \\
\hline $\mathrm{C}_{4} \mathrm{BH}$ & 20 & & & & & & & & & & & & & & & & & & & .0 \\
\hline $\mathrm{C}_{A B H}$ & 10 & & & & & $\begin{array}{l}0.0 \\
17\end{array}$ & & & & & & 78 & & & & 0.0 & & & & \\
\hline $\mathrm{CPH}$ & 30 & & & 50 & & & & & & & & & & & & & & & & \\
\hline $\mathrm{C}_{5} \mathrm{BH}$ & & & & & & & & & & & & & & & & & & & & 1 \\
\hline${ }_{B} \mathrm{BH}$ & 60 & & & & & & & & & & & 44 & & & & $\begin{array}{c}0.0 \\
14\end{array}$ & & & 45 & 0.1 \\
\hline $\mathrm{C}_{5} \mathrm{BH}$ & & & & & & & & & & & & & & & & 0.0 & & & .0 & .1 \\
\hline & 30 & & & $\begin{array}{l}0.0 \\
00\end{array}$ & $\begin{array}{c}0.0 \\
00\end{array}$ & 07 & & & 00 & & & 1 & & & & $\begin{array}{c}0.0 \\
00\end{array}$ & $\begin{array}{c}0.8 \\
12 \\
\end{array}$ & & 60 & $\begin{array}{l}0.1 \\
76 \\
\end{array}$ \\
\hline & & & & & & & & & & & & & & & & & & & & .3 \\
\hline & & & & & & & & & & & & & & & & & & & & \\
\hline & & 170 & & $\begin{array}{l}1.9 \\
20\end{array}$ & & & & & & & & & & & & & & & & \\
\hline
\end{tabular}

Tables 2 shows the mean concentration of metal cations measured in study cells whereas Table 3 shows the values for the hydrogeochemical attributes (x-values) used for the simulation.

Table 2: mean concentration of metal cations in each study cell

\begin{tabular}{|c|c|c|c|c|c|c|c|c|c|c|}
\hline Study cell & arsenic & lead & cupper & chromium & cobalt & nickel & manganese & Iron & cadmium & Mercury \\
\hline C1 & 0.000 & 0.213 & 0.016 & 0.691 & 0.128 & 0.019 & 0.210 & 0.377 & 0.088 & 0.755 \\
\hline C2 & 0.013 & 0.454 & 0.097 & 1.099 & 0.275 & 0.025 & 0.648 & 0.393 & 0.059 & 0.424 \\
\hline C3 & 0.013 & 0.374 & 0.013 & 0.000 & 0.026 & 0.011 & 0.514 & 0.008 & 0.031 & 0.609 \\
\hline C4 & 0.018 & 0.640 & 0.177 & 0.697 & 0.438 & 0.040 & 0.573 & 0.429 & 0.064 & 1.042 \\
\hline C5 & 0.000 & 0.563 & 0.022 & 0.062 & 0.004 & 0.008 & 0.580 & 0.246 & 0.082 & 0.707 \\
\hline C6 & 0.017 & 0.981 & 0.022 & 2.069 & 0.004 & 0.000 & 0.792 & 0.021 & 0.144 & 0.738 \\
\hline C7 & 0.000 & 0.364 & 0.775 & 0.232 & 1.300 & 0.118 & 0.220 & 2.105 & 0.032 & 1.450 \\
\hline C8 & 0.000 & 0.888 & 0.981 & 0.607 & 1.598 & 0.154 & 0.207 & 2.905 & 0.025 & 0.111 \\
\hline
\end{tabular}

Table 3: Values for the hydrogeochemical attributes in the study cells. 
Citation: Emereibeole EI, et al., Predictive Model for Leachate Contamination of Groundwater around a municipal Waste Dump: Case Study of Obosi, Southeastern Nigeria. Australian Journal of Basic and Applied Sciences, 13(6): 115-121.DOI: 10.22587/ajbas.2019.13.6.13

\begin{tabular}{|c|c|c|c|c|c|c|c|c|}
\hline \multirow{2}{*}{ Parameters } & \multicolumn{8}{|c|}{ Cells } \\
\hline & C1 & C2 & C3 & C4 & C5 & C6 & C7 & C8 \\
\hline $\begin{array}{l}\text { Distance of Boreholes from dumpsite }(\mathrm{m}) \\
\qquad\left(\mathrm{x}_{1}\right)\end{array}$ & 551.50 & 1051.87 & 1551.40 & 2051.60 & 2551.32 & 3051.22 & 3551.06 & 4051.66 \\
\hline Vadose conductivity (m/day) $\left(\mathrm{x}_{2}\right)$ & 32.85 & 32.85 & 32.85 & 32.85 & 32.85 & 32.85 & 32.85 & 32.85 \\
\hline Borehole depth $(\mathrm{m})\left(\mathrm{x}_{3}\right)$ & 45.75 & 47.28 & 61.00 & 76.25 & 48.80 & 67.10 & 91.50 & 54.90 \\
\hline Net Recharge (m/day) $\left(\mathrm{x}_{4}\right)$ & 1.45 & 1.45 & 1.45 & 1.45 & 1.45 & 1.45 & 1.45 & 1.45 \\
\hline Hydraulic head $(\mathrm{m})\left(\mathrm{x}_{5}\right)$ & 6.15 & 10.68 & 3.40 & 13.50 & 24.54 & 9.90 & 18.47 & 67.24 \\
\hline
\end{tabular}

The $\mathrm{k}$ values generated after solving the polynomial equations are shown in table 4 .

Table 4: Predictive constants obtained from solving pollutant regression equations

\begin{tabular}{|c|c|c|c|c|c|c|}
\hline \multirow{2}{*}{ Pollutant } & \multicolumn{7}{|c|}{ Predictive constants } \\
\cline { 2 - 7 } & $\mathrm{k}$ & $\mathrm{k}_{1}$ & $\mathrm{k}_{2}$ & $\mathrm{k}_{3}$ & $\mathrm{k}_{4}$ & $\mathrm{k}_{5}$ \\
\hline Arsenic & 0.000 & $9.0 \times 10^{-7}$ & $2.368 \times 10^{-4}$ & $3.91 \times 10^{-5}$ & 0.000 & $-2.332 \times 10^{-4}$ \\
\hline Lead & 0.000 & 0.0003 & 0.0188 & -0.0092 & 0.000 & -0.0050 \\
\hline Cupper & 0.000 & -0.0001 & -0.0278 & 0.0173 & 0.000 & 0.0218 \\
\hline Chromium & 0.000 & -0.0278 & 2.4205 & 0.2090 & 0.000 & 1.8297 \\
\hline Cobalt & 0.000 & -0.0003 & -0.0478 & 0.0332 & 0.000 & 0.0397 \\
\hline Nickel & 0.000 & 0.000 & -0.0040 & 0.0029 & 0.000 & 0.0037 \\
\hline Manganese & 0.000 & 0.0002 & 0.0290 & -0.0114 & 0.000 & -0.0150 \\
\hline Iron & 0.000 & -0.0004 & -0.0643 & 0.0432 & 0.000 & 0.0644 \\
\hline Cadmium & 0.000 & 0.000 & 0.0051 & -0.0024 & 0.000 & -0.0028 \\
\hline mercury & 0.000 & 0.000 & -0.0086 & 0.0199 & 0.000 & -0.0066 \\
\hline
\end{tabular}

Substituting these $\mathrm{k}$ values into the regression equation for each metal cation gives equations 4.0 to 4.9 below, which are model expressions for prediction of pollution concentration of metallic ionic moieties.

$\mathrm{y}_{\mathrm{As}}=9.0 \times 10^{-7} \mathrm{x}_{1}+2.368 \times 10^{-4} \mathrm{x}_{2}+3.91 \times 10^{-5} \mathrm{x}_{3}-2.332 \times 10^{-4} \mathrm{x}_{5}$

(4.0)

$\mathrm{y}_{\mathrm{Pb}}=0.0003 \mathrm{x}_{1}+0.0188 \mathrm{x}_{2}-0.0092 \mathrm{x}_{3}-0.0050 \mathrm{x}_{5}$

$\mathrm{y}_{\mathrm{Cu}}=-0.0001 \mathrm{x}_{1}-0.0278 \mathrm{x}_{2}+0.0173 \mathrm{x}_{3}+0.0218 \mathrm{x}_{5}$

$\mathrm{y}_{\mathrm{Cr}}=-0.0278 \mathrm{x}_{1}+2.4205 \mathrm{x}_{2}+0.2090 \mathrm{x}_{3}-1.8297 \mathrm{x}_{5}$

$\mathrm{y}_{\mathrm{Co}}=-0.0003 \mathrm{x}_{1}-0.0478 \mathrm{x}_{2}+0.0332 \mathrm{x}_{3}+0.0397 \mathrm{x}_{5}$

$\mathrm{y}_{\mathrm{Ni}}=-0.004 \mathrm{x}_{2}+0.0029 \mathrm{x}_{3}+0.0037 \mathrm{x}_{5}$

$\mathrm{y}_{\mathrm{Mn}}=0.0002 \mathrm{x}_{1}+0.0290 \mathrm{x}_{2}-0.0114 \mathrm{x}_{3}-0.0150 \mathrm{x}_{5}$

$\mathrm{y}_{\mathrm{Fe}}=-0.0004 \mathrm{x}_{1}-0.0643 \mathrm{x}_{2}+0.0432 \mathrm{x}_{3}+0.0644 \mathrm{x}_{5}$

$\mathrm{y}_{\mathrm{Cd}}=0.0051 \mathrm{x}_{2}-0.0024 \mathrm{x}_{3}-0.0028 \mathrm{x}_{5}$

$\mathrm{y}_{\mathrm{Hg}}=-0.0086 \mathrm{x}_{2}+0.0199 \mathrm{x}_{3}-0.0066 \mathrm{x}_{5}$

\section{DISCUSSION}

\subsection{Influence of $k$ Values on the Contribution of Metal Ions to Pollution}

The negative $\mathrm{k}_{1}$ values for $\mathrm{Cu}^{2+}, \mathrm{Cr}^{3+}, \mathrm{Co}^{2+}$ and $\mathrm{Fe}^{2+}$ in Table 4 show that as the distance increases, the contribution to pollution of these cations decrease (Mor et al., 2006). $\mathrm{Ni}^{+}, \mathrm{Cd}^{2+}$, and $\mathrm{Hg}^{2+}$ contribution to pollution is not distance dependent. $\mathrm{Pb}^{2+}$ and $\mathrm{Mn}^{2+}$ which have positive $\mathrm{k}_{1}$ values show that their contribution to pollution increase with distance. This is an indication of extraneous contamination that is not leachate in origin (Jagloo, 2002). $\mathrm{Cu}^{2+}, \mathrm{Co}^{2+}, \mathrm{Ni}^{+}, \mathrm{Fe}^{2+}$ and $\mathrm{Hg}^{2+}$ have negative $\mathrm{k}_{2}$ which indicates that conductivity of the vadose region decreases their contribution to pollution. $\mathrm{Pb}^{2+}, \mathrm{Mn}^{2+}$ and $\mathrm{Cd}^{2+}$ contribution to pollution decrease with depth of borehole (Chove, 2018). The $\mathrm{k}_{4}$ which represents coefficient of net recharge does not influence the concentration of metal ions. This is expected as the ionic concentrations are diluted or concentrated at equal rates. Furthermore, $\mathrm{k}_{5}$ which is the coefficient of hydraulic head is negative for $\mathrm{Pb}^{2+}, \mathrm{Mn}^{2+}, \mathrm{Cd}^{2+}$ and $\mathrm{Hg}^{2+}$. Negative $\mathrm{k}_{5}$ values indicate less contribution of these cations to aquifer pollution, corresponding to increase in the hydraulic head (Mkude, 2015).

\subsection{Model Justification}

In order to verify the applicability of the simulation equations, four independent boreholes were sampled in the study cells and analyzed as against values from simulation. These values are shown in Table 5.

Table 5: Simulated and observed values for groundwater contaminants in the study area

\begin{tabular}{|c|c|c|c|c|c|c|c|c|}
\hline \multirow{2}{*}{ Parameters } & \multicolumn{4}{|c|}{ Simulated Values } & \multicolumn{4}{c|}{ Measured Values } \\
\cline { 2 - 9 } & C2BH1 & C3BH2 & C4BH3 & C5BH4 & C2BH1 & C3BH2 & C4BH3 & C5BH4 \\
\hline Arsenic & 0.009 & 0.009 & 0.011 & 0.008 & 0.006 & 0.000 & 0.038 & 0.016 \\
\hline Lead & 0.325 & 0.307 & 0.563 & 0.284 & 0.251 & 0.519 & 0.345 & 0.313 \\
\hline Copper & 0.034 & 0.314 & 0.063 & 0.945 & 0.057 & 0.009 & 0.057 & 0.051 \\
\hline
\end{tabular}


Citation: Emereibeole EI, et al., Predictive Model for Leachate Contamination of Groundwater around a municipal Waste Dump: Case Study of Obosi, Southeastern Nigeria. Australian Journal of Basic and Applied Sciences, 13(6): 115-121.DOI: 10.22587/ajbas.2019.13.6.13

\begin{tabular}{|c|c|c|c|c|c|c|c|c|}
\hline Chromium & 0.534 & 0.357 & 0.893 & 0.000 & 1.553 & 0.215 & 0.367 & 0.844 \\
\hline Cobalt & 0.160 & 0.642 & 0.104 & 1.726 & 0.082 & 0.002 & 0.008 & 0.073 \\
\hline Nickel & 0.039 & 0.094 & 0.062 & 0.216 & 0.000 & 0.167 & 0.027 & 0.000 \\
\hline Manganese & 0.414 & 0.288 & 0.540 & 0.000 & 0.772 & 0.170 & 0.270 & 0.120 \\
\hline Iron & 0.220 & 0.882 & 0.115 & 0.439 & 0.224 & 1.425 & 0.000 & 0.139 \\
\hline Cadmium & 0.028 & 0.000 & 0.009 & 0.000 & 0.020 & 0.022 & 0.009 & 0.039 \\
\hline Mercury & 0.667 & 0.946 & 0.878 & 1.393 & 0.176 & 0.157 & 0.159 & 0.099 \\
\hline
\end{tabular}

The pertinent data for leachate migration simulations are presented in Table 6.

Table 6: values for the contaminant migration attributes in Boreholes

\begin{tabular}{|c|c|c|c|c|}
\hline Parameters & \multicolumn{4}{|c|}{ Borehoes and Cells } \\
\cline { 2 - 5 } & C2BH1 & C3BH2 & C4BH3 & C5BH4 \\
\hline Distance of Boreholes from dumpsite $(\mathrm{m})\left(\mathrm{x}_{1}\right)$ & 671.20 & 1121.22 & 1742.12 & 2062.33 \\
\hline Vadose conductivity $\left(\mathrm{m} /\right.$ day) $\left(\mathrm{x}_{2}\right)$ & 32.85 & 32.85 & 32.85 & 32.85 \\
\hline Borehole depth $(\mathrm{m})\left(\mathrm{x}_{3}\right)$ & 50.00 & 65.00 & 60.00 & 91.50 \\
\hline Net Recharge $\left(\mathrm{m} /\right.$ day) $\left(\mathrm{x}_{4}\right)$ & 1.45 & 1.45 & 1.45 & 1.45 \\
\hline Hydraulic head $(\mathrm{m})\left(\mathrm{x}_{5}\right)$ & 6.85 & 9.85 & 5.15 & 22.10 \\
\hline
\end{tabular}

'The one-way ANOVA test revealed that the mean concentrations of all the cations measured differed significantly across the boreholes studied $(\mathrm{Sig} F$ values $=$ As 0.005, Pb 0.000, Cu 0.000, Cr 0.000, Co 0.000, Ni 0.000, Mn 0.037, Fe 0.000, Cd 0.000 and $\mathrm{Hg} 0.000)$ at $\mathrm{p}<0.05$.

Furthermore, the pair-wise student's t-test show that except for $\mathrm{Hg}$, the simulated and measured values of the other cations studied were not significantly different at the $95 \%$ confidence level (sig. $\mathrm{t}=0.026$ ). This result indicates that at $95 \%$ confidence level, the simulation becomes invalid.

\section{CONCLUSION}

A statistical comparison applying ANOVA and student's t-tests show that the model was not more than $95 \%$ accurate. The net recharge was shown by the numerical analysis to be insignificant as a pollution factor. Furthermore, the simulations indicate that as radial distance from the landfill increases, concentration of contaminants in groundwater decreases (Anne, 1993). The model can be modified by including an error factor which can be evaluated by comparing two models of similar characteristics with varying factors.

\section{ACKNOWLEDGEMENT}

We greatly appreciate the assistance of the Geographical laboratory unit, Department of Geophysics, Pollution control and Geo-environmental hazards laboratory units, Federal University of Technology, Owerri, for all the technical assistance received from them during the course of this study.

\section{REFERENCES}

Abu-Rukah, Y., and O. Kofahi, 2001. The Assessment of The Effect of Landfill Leachate on Groundwater Quality. A Case Study of El-Akader Landfill Site-North Jordan. Arid Environment, 49: 615-630. Doi:10.1006/jare.2001.0796

Al-Khadi, S., 2006. Assessment of Groundwater Contamination Vulnerability in the Vicinity of Abqaiq Landfill-A GIS Approach, Doctoral Dissertation, King Fahd University of Petroleum and Minerals, Saudi Arabia

American Public Health Association (APHA), American Water Works Association (AWWA), and Water Environment Federation (WEF), 1999. Standard Methods for the Examination of Water and Wastewater, 20th Edition on CD

Anne, J., and G. Fred, 1993. Groundwater Pollution by Municipal Landfill: Leachate Composition, Detection and Water Quality Significance. In the Proceedings of October 1993 4th International Landfill Symposium, Sardinia, Italy, Pp: 1093-1103

Chove, L. M., 2018. Effect of Depth and Distance of the Borehole from the Septic Tank on the Physico-chemical Quality of Water. Journal of Food Studies, 7(1): 41-55. Doi:10.5296/jfs.v7i 1.12187

Christensen, T., and P. Kjeldsen, 2001. Biogeochemistry of Landfill Leachate Plumes. Applied Geochemistry.16, pp. 659-718. Doi:10.1016/50883-2927(00)00082-2

De Rosa, E., D. Rubel, M. Tudino, A. Viale, and R. Lombardo, 1996. The Leachate Composition of an Old Waste Dump Connected to Groundwater: Influence of the Reclamation Works. Environmental Monitoring Assessment, 40: 239 - 252. Doi:10.1007/BF00398869

Egboka, B., J. Cherry, R. Farvolden, and E. Frind, 1983. Migration of Contaminants in Groundwater at a Landfill: A Case Study, 3. Tritium as an Indicator of Dispersion and Recharge. In: Cherry, J. (Guest-Editor). Migration of Contaminants in Groundwater at a Landfill: A Case Study. Journal of Hydrology, 63: 51-80. Doi:10.1016/0022-1694(83)90222-6

Erses, A., M. Fazal, T. Onaya, W. Craig, 2005. Determination of Solid Waste Sorption Capacity for Selected Heavy Metals in Landfills. Journal of Hazardous Materials, B (121):223-32 doi:10.1016/j.jhazmat.2005.02.011 
Ezeabasili, A., O. Anike, and B. Okoro, 2015. Urban Water Pollution by Heavy Metals and Health Implication in Onitsha, Nigeria. African Journal of Environmental Sciences and Technology,9(4):325-331. Doi:10.5897/AJEST2013.1587

Ezeabasili, A., B. Okoro, and A. Okonkwo, 2014. Assessment of Ground Water Quality in Awka, Anambra State, Nigeria. International Journal of Science and Technology, 3(3): 81-93. Doi:10.4314/stech.v3i3.8

Fatta, D., A. Padadopoulos, and M. Loizidou, 1999. A Study on the Landfill Leachate and its Impact on the Groundwater Quality of the Greater Area. Environmental Geochemical Health, 21 (2): 175 - 190. Doi: 10.1023/A:1006613530137

Flyhammar, P., 1997. Estimation of Heavy Metals Transformation in Municipal Solid Waste. The Science of the Total Environment, 198(2): 123 - 133. Doi:10.1016/50048-9697(97)05439-9

Hudak, P., 1998. Groundwater Monitoring Strategies for Variable Versus Constant Contaminant Loading Functions. Environmental Monitoring Assessment, 50(3): 271- 288. Doi:10.1023/A:1005806330741

Jagloo, K., 2002. Groundwater Risk Analysis in the Vicinity of a Landfill, A Case Study in Mauritius, M.Sc. thesis, Royal Institute of Technology, Stockholm

Jhamnani, B., and S. Singh, 2009. Groundwater Contamination Due to Bhaswa Landfill Site in New Delhi. International Journal of Civil and Environmental Engineering, 1(3): 121-125

Mkude, I. T., 2015. Comparative Analysis of Heavy Metals From Groundwater Sources Situated in Keko and Kigogo Residential Areas, Dares Salaam. Journal of Water Resources and Ocean Science, 4(1): 1-5. Doi:10.11648/j.wros.20150401.11

Moo-Yound, H., A. Johnson, D. Carson, C. Lew, S. Liu, and K. Hancock, 2004. Characterisation of Infiltration Rates from Landfills: Supporting Groundwater Modeling Effort. Environmental Monitoring Assessment, 96(1-3): 283 - 311

Mor, S., Ravindra, K., Dahiya, R. P. \& Chandra, A. (2006). Leachate characterization and assessment of groundwater pollution near municipal solid waste landfill site. Environmental Monitoring Assessment, 4,pp. 325-334. Doi:10.1007/s10661-0061505-7

Ogbukagu, I., 1986. Water Supply of Njikoka and Awka Areas of Anambra State. Journal of Earth Science, 5:511-519

Pariatamby, A. and M. Tanaka, 2013. Municipal Solid Waste Management in Asia and the Pacific Islands: Challenges and Strategic Solutions. Singapore: Springer Science and Business Media. Retrieved from https://books.google.com.ng/books?id=nRXHBAAAQBAJ

Renou, S., J. Givaudan, S. Poulain, F. Dirassouyan, and P. Moulin, 2008. Landfill Leachate Treatment: Review and Opportunity. Journal of Hazardous Materials, 150 (3): 468-493. Doi:10.1016/j.jhazmat.2007.09.077

Saarela, J., 2003. Pilot Investigation of Surface Parts of Three Closed Landfills and Factors Affecting them. Environmental Monitoring Assessment, 84(1-2): 183 - 192. Doi:10.1023/A:1022859718865

Salami, L., O. A. Olafadehan, G. Babagana, and A. A. Susu, 2013. Prediction of Concentration Profiles of Contaminants in Groundwater Polluted by Leachates from a Landfill Site. International Journal of Recent Research and Applied Studies, 15(3): 365-378

Stoline, M., R. Passerp, and J. Booker, 1995. Clay Barrier Systems for Waste Disposal Facilities. E and FN Spon, London, United Kingdom

Thirumalaivasan D., 2001. Aquifer Vulnerability Assessment Using Analytic Hierarchy Process and GIS for Upper Palar Watershed, Centre for Remote Imaging and Sensing and Processing (CRISP), National University of Singapore 